\title{
HOW POLYCYSTIC OVARY SYNDROME (PCOS) AFFECTS THE FERTILITY POTENTIAL OF REPRODUCTIVE AGE FEMALES: REVIEW
}

\author{
Muhjah Falah Hassan ${ }^{1} \&$ Rabab Zahir Al-yasiry ${ }^{2}$ \\ 'Department of Anatomy and Embryology, Faculty of Medicine, Kerbala University ,Iraq. \\ ${ }^{2}$ Department of Anatomy and Histology, Faculty of Medicin, Babylon University, Iraq.
}

Corresponding author: doctor89muhjah@yahoo.com

\begin{abstract}
Polycystic ovary syndrome (PCOS) is one of the major causes of decreased fertility in young females. Multiple extra- and intra-ovarian factors which are worked together or separately are responsible for sub-fertility. However, still, ovulatory dysfunction is the cornerstone that occurs as a result of hormonal dysfunction and consequently affects oocytes' quality, embryonic development and finally, implantation failure with a significant number of females seek assisted reproduction for getting pregnancy.
\end{abstract}

Keywords: PCOS, Sub-fertility, Hormonal dysfunction, Implantation failure and Assisted reproduction. 


\section{Introduction}

Polycystic ovary syndrome is a condition of chronic anovulation which usually occurs as a result of an imbalance of reproductive hormones. Women with PCOS produce abnormally high androgen level which leads to failure of ovulation, skipping of menstrual period and difficulty in getting a pregnancy(Mahesh et al., 2018). Ovarian dysfunction represented by anovulation plays an important role in decreasing the fertility potential of the affected females (Adam, 2014). However, multiple intra and extra ovarian factors could be considered (Josefin et al., 2017).

So, how PCOS decrease the conception chance is a matter of debate and how could be bypass these factors is a matter of wide research.

\section{Definition, Diagnosis and Clinical Presentations of PCOS}

The definition of the PCOS has been much debated. The main pathophysiological abnormalities of the syndrome tend to be multi-factorial and poly-genic. Still ovarian dysfunction plays a central role in the pathophysiology despite multiple extra-ovarian aspects (Adam, 2014)

At a joint European Society of Human Reproduction and Embryology (ESHRE) / American Society for Reproductive Medicine (ASRM) consensus meeting in Rotterdam in 2004, PCOS was diagnosed upon finding two from three criteria: 1. oligo and/or anovulation 2 . clinical $+/-$ biochemical sings of androgen excess and 3. polycystic ovaries by ultrasound, After the exclusion of other etiologies (Fauser et al., 2012).

The morphological features of polycystic ovary also were defined as an increased ovarian volume more than 10 cm3 and/or thickened stroma and appearance of 12 or more follicles measuring 2-9 $\mathrm{mm}$ in diameter distributed peripherally. Either one or both ovaries may be involved (Fulghesu et al.,2017).

There is a variability in the expression of the syndrome among the patients and for a patient the sign and symptoms might be changed with time (Mojgan, 2017). It may be clinically silent and the expression of it can be enhanced by multiple factors.
Increased weight is thought to be the most predominant one. However, clinical and biochemical factors which define PCOS may not be exhibited in all women with polycystic ovaries. These are represented by disturbances of menstrual cycle, hirsutism, acne and alopecia; and biochemical abnormalities in form of increased concentrations of $\mathrm{LH}$, testosterone and androstenedione in the serum. Hyper-insulinaemia and obesity may associate although, only $40 \%-50 \%$ of females with polycystic ovary are overweight (Adam, 2014)

Polycystic ovary syndrome presentation is usually variable in which either one, all, or a combination of the previously mentioned features can appear together with a polycystic ovaries picture during trans vaginal ultrasound examination (Fritz \& Speroff, 2012).

Weight gain is associated with worsening of symptoms, whereas weight reduction enhances the endocrine, metabolic profile and the symptoms of the syndrome (Josefin et al., 2017). Although weight loss does not necessarily normalize the condition, it mainly improves the response to therapy, either for sub-fertility or for other abnormalities associated with the syndrome.

The endocrine profile that many believe to be a diagnostic is elevated serum level of $\mathrm{LH}$, testosterone and androstenedione together with normal or lower normal serum level of $\mathrm{FSH}$ and abnormal E2 production. (Sudha et al., 2017).

A non-invasive ultrasound scan is used for the assessment of total ovarian volume and morphology. The most relevant one that is used for the diagnosis of polycystic ovaries is trans-vaginal ultrasound (TVUS). More recently, the threshold number of follicles should be 19 and serum concentration of anti-mullerian hormone $(\mathrm{AMH})$ is greater than $35 \mathrm{pml} / \mathrm{L}$ which is more precise than ultrasound features(Yahia et al., 2013).

So, the diagnosis is usually restricted for those females who show polycystic ovaries picture by ultrasound and display one clinical symptom or more with or without one or more biochemical abnormalities after the exclusion of certain underlying diseases that might 
produce similar biochemical and ultrasound features such as pituitary and adrenal problems (Arian et al., 2015).

\section{Prevalence of PCOS}

The exact prevalence of PCOS is difficult to be determined definitively within the general population. It may show a considerable variation among the studied populations (Amir et al., 2016).

Those who are selected upon the presence of clinical symptoms could be expected to have a greater prevalence than that exists in the general population. An estimated prevalence is approximately $4-8 \%$, but it may be as high as $25 \%$ in some populations (Susie et al., 2018). With increased rates and severity of symptoms in South-East Asian women. Around 22-33 \% of women will show polycystic ovaries on ultrasound scan with no any associated symptom. Those tend to be considered as normal variant and do not require further investigations or interventions (Teede et al., 2010).

\section{PCOS and Infertility}

Sub-fertility is a frequent complaint in PCOS women and usually occurs as a result of anovulatory cycle due to the failure of normal ovarian function (Kini, 2012).

How PCOS affects the fertility potential of the female is a controversial topic and usually results from multiple intra and extra ovarian factors which work together or separately and end in sub-fertility. Despite multiple interlacing causes of sub-fertility in PCOS women, ovulatory dysfunction is considered to be the most common cause. In fact, approximately $75 \%$ of all cases of anovulatory infertility have PCOS (Fritz \& Speroff, 2012).

It has been showed that the effect of PCOS which may result in sub-fertility acts at multiple levels : central hypothalamic-pituitary-ovarian axis ) results in anovulation, intra ovarian which adversely affects the quality of oocytes which in turn affects the quality of the resulting embryos or intrauterine level results in implantation failure and recurrent pregnancy loss (Susie et al., 2018).

\section{PCOS and Chronic Anovulation}

The precise mechanism is still not known but anovulation in PCOS women most likely results from the inappropriate secretion of gonadotropins and change in the pulsatile secretion of $\mathrm{GnRH}$ which leads to excessive production of $\mathrm{LH}$ relative to $\mathrm{FSH}$. Whether the primary cause of PCOS is the hypothalamus or PCOS occurs secondary to abnormality in steroid hormones feedback is a matter of debate (Hoffman et al., 2012). LH stimulates ovarian androgen production while the insufficient amount of $\mathrm{FSH}$ interferes with normal granulose cells aromatase activity and impairs androgen conversion to E2 (Sudha et al., 2017). Excessive circulating androgens also will enhance the aromatase conversion activity to produce E2 which is responsible for the chronic negative feedback to hypothalamus and pituitary gland in comparison to the normal fluctuations in the feedback which are observed in normal women as a result the of a dominant follicle presence and rapid change in E2 levels.

Insulin resistance might be considered as a potent contributor to follicular atresia in polycystic ovaries. Impairment of normal follicular development leads to anovulation and subsequent oligo/amenorrhea in those patients (Hoffman et al., 2012).

\section{Effect of PCOS on Oocyte Development}

As a result of dysfunctional ovarian steroid-genesis which is the main pathophysiological abnormalities in PCOS women, theca cells from those patients will show an 1increased androgen secretion when they are cultured separately in vivo (Walters \& Handelsman, 2016). This state of hyper androgenaemia will alter the microenvironment within the follicles leading to impaired folliculogenesis (Yan et al., 2016).

It might be possible that an elevated insulin level may also act as a contributing factor to excessive androgen production and will negatively affect proper oocyte development. High androgen level has a deleterious effect on folliculogenesis ends in follicular atresia. Which tends to be 
caused by androgens entrance into the granulose cells of pre-antral follicles, binding to intracellular receptors and leading to cellular apoptosis. The hyper androgenic state also leads to disturbed meiotic division as well as mitotic division of the oocytes and in turn results in improper maturation of the oocytes and developmental compromise (Yan et al., 2016).

Maturation defects of the oocytes in the polycystic ovary are markedly still uncharacterized. There are inhibitory signals that hold meiosis in prophase $\mathrm{I}$ in the normal ovary (Satin \& Rruce, 2008). Successful ovulation to be occurred needs sufficient amounts of gonadotropins. Both $\mathrm{LH}$ and $\mathrm{FSH}$ are necessary for enhancement of follicular growth and secretion of E2 and testosterone within a threshold level to oppose the inhibitory signals and allow oocytes initiate and complete meiosis.

Excess androgen in PCOS stimulates multiple ovarian follicles in an equivalent time thus prevents the development of a single dominant follicle and leads to ovulation failure and relative resistance to gonadotropins (Dumesic et al., 2015). Intrinsic molecular defects within oocytes may also play a role.

Despite the abundant number of oocytes within polycystic ovaries, these oocytes have a reduced fertilizing ability and this leads to a decreased fertility in PCOS patients and makes fertility treatment challenging (Okohue et al., 2013).

A raised concentration of $\mathrm{LH}$, together with premature LH surge, may have an impact on follicle and oocyte development and are believed to be responsible for lowering pregnancy rate in those patients.

A long time exposure of oocytes to high LH concentrations also leads to impairment of cytoplasmic maturity of oocytes. Therefore it can be postulated that a prolonged pituitary suppression could be helpful in PCOS patients.

\section{Effect of PCOS on Embryo Quality}

The quality of an embryo to a great extent depends on the quality of oocyte from which it was obtained. Embryos with higher cell numbers, regular appearing cells, and little or no fragmentation have a higher overall chance of implantation (Mina, 2013).

In PCOS patients, the increasing level of androgen leads to a decreased ovum potential to produce top-quality embryo.

A high serum androgen level also has a negative impact on the quality of embryo itself and its quality gradually deteriorated with increased patients' age. The embryos of PCOS women with high serum androgen and an age more than 35 years old, are usually at an increased risk of developmental arrest (Baoli et al., 2015). PCOS women with hyper androgenemia and obese women had shown a delayed early embryonic developmental stages. Embryos which were derived from such women were characterized by delayed two pronuclus (2PN) formation post injection and delayed 4 cell stage up to 8 cell stage development (Wissing et al., 2014). But this delay in the embryonic development had no significant bad impact on implantation and pregnancy rate (Bellver et al., 2013). This will strengthen the concept that both the metabolic and hormonal state of PCOS women will lead to derangement of early embryonic development with no significant clinical outcome (Fancsovits et al.,2011). The effect of PCOS on the embryo quality tends to be diverse as PCOS women with normal androgen level still have an ability to produce developmentally normal embryos in synchronization with post injection time (Bellver et al., 2013).

The number of embryos which are produced by lab fertilization in such women are more when compared to women with normal ovary due to more oocytes which are produced. Since the chance of a good quality embryo development would be increased and during ARTs, the probability of successful fertilization and successful conception is increased because of the ability of choosing oocytes and embryos with a good quality from all retrieved oocytes 
and formed embryos (Boomsma et al., 2006).

\section{Effect of PCOS on Implantation of Embryo and Recurrent Implantation Failure}

Implantation involves the adhesion of the embryo to the decidual layer of uterine endometrium with descent toward the basement membrane and its invasion to the stroma. This process is regulated by a complex system that involves interaction of hormones, growth factors, adhesion molecules, extracellular matrices and prostoglandins.

Although ARTs are able to achieve fertilization in 70-80\% of cases, the average live birth rate remains only 30$40 \%$. This could be explained by reduced endometrial receptivity and implantation failure (Apostolos, 2018).

The incidence of miscarriage in PCOS patients is $30-40 \%$ which is three times higher than normal women. The mechanisms by which PCOS causes a miscarriage are believed to be as a result of elevated androgen, elevated $\mathrm{LH}$, increased insulin secretion and excess body weight (Apostolos, 2018).

Impairment of endometrial blood flow, growth factors, adhesive molecules and cytokines are also likely to be candidates (Cakmak \& Taylor, 2011).

Several studies suggested a link between hyper secretion of $\mathrm{LH}$ and early miscarriage. How an elevated LH concentration contributes to miscarriage till now is a matter of debate. A raised follicular level of LH can cause premature maturation of oocytes, release a physiologically aged oocytes and make viable embryos therefore unlikely to be produced (Revelli et al., 2009).

Also elevated LH concentration can lead to impairment of endometrial receptivity and hence implantation failure, This was based on the discovery of LH receptors on the endometrium (Duru et al., 2004). Although recent studies have showed that there is no correlation between elevated LH and implantation failure, so depending on authors opinion, hyper secretion of LH is less likely responsible for making other endocrine and local factors to be considered instead(Sudha et al., 2017).

The mechanism by which high serum androgen level can cause implantation failure in PCOS patients is mainly related to its bad effect on the normal uterine endometrial development (Cocksedge et al., 2008). This concept depends on the fact that women with high androgen level have a reduced expression of the endometrial protein; placental protein (PP14) (glycodelin A) during the periimplantation period. In addition high androgen level also may have an adverse effect on the quality of oocytes and hence embryo viability and implantatio.

Furthermore, since hyper androgenism and hyper insulinaemia are usually accompanied to each other in PCOS patients, hyper androgenism may indirectly affect insulin secretion via its effect on IGF-1 receptors. The mechanism by which miscarriage may result is unclear. Possibly, a raised insulin level is responsible for the decreasing serum glycodelin and insulin like growth factor -1 binding protein (IGFBP-1) concentrations during the $1^{\text {st }}$ trimester in PCOS pregnant patients (Stepto et al., 2013). Low levels of these proteins leads to insufficient endometrial development during the peri-implantation period. Two alternative mechanisms might also be responsible. Firstly ; insulin resistance may lead to transport of excess amounts of glucose from the mother to the fetus resulting in the first trimester fetal loss.

Secondly, It was believed that there is a link between raised insulin concentrations and raised plasminogen activator inhibitor-1 (PAl-1) concentrations. This will lead to placental insufficiency via increased thrombosis.

Glycodelin considered to be an important promising indicator of implantation. It plays a role in the processes pertaining to endometrial receptivity and implantation. Its low levels during the mid-cycle fertile window are conducive to fertilization, while the higher levels during the midluteal implantation window (between day 19 and day 24 of the 28-day cycle) allow for implantation to take place. It has been demonstrated that the expression of glycodelin is increased in the endometrium during the implantation 
window and late luteal phase. Low serum and endometrial levels of glycodelin are observed in anovulatory cycles. This will lead to the impairment of endometrial functions making the pre-implantation environment is less suitable for implantation (Sudha et al., 2017). Thus, low glycodelin level leads to implantation failure, $1^{\text {st }}$ trimester pregnancy loss, and recurrent pregnancy loss (Selda et al., 2015).

\section{Conclusions}

Multiple intra and extra ovarian factors can affects the reproductive potentials in PCOS women; increased body weight, hyper androgenemia, insulin resistance and excess $\mathrm{LH}$ production which may lead to failure of normal ovulation, alter the micro-follicular environment leading to produce bad quality oocytes and embryos and decrease uterine receptivity to the developed embryos which in turn decrease the chance of the women to get a spontaneous pregnancy.

\section{References}

Adam, H. (2014). Polycystic Ovary Syndrome, Management-Diagnosis and Treatment. In : Infertility in practice. $4^{\text {th }}$ ed. CRC Press is an imprint of Taylor \& Francis Group, an Informa business, 201236.

Amir, H., Hossein, M., \& Anoosh, N. (2016). Comparison of the levels of $\mathrm{LH}$, $\mathrm{FSH}$, TSH, Prolactin, Progesterone and Estradiol hormones between Iranian infertile women with PCOS and healthy women. Int. J. of Medical Research and Health Sciences.,5(12):370-375.

Apostolos, T. (2018). Polycystic ovary syndrome and early pregnancy loss : A review article. EC Gynecology.,7(2) : 3542.

Arain, F., Arif, N., \& Halepota, H. (2015). Frequency and outcome of treatment in polycystic ovaries related infertility. Pak Journal Medical Sci.,31(3):694-699.

Baoli, Y., Haoying, H., Duo, W., Xiaobing, S., Juanke, X., \& Cuilian, Z. (2015). Patients with polycystic ovary syndrome have a successful embryo arrest. Int. J. Clin. Exp. Med.,8(4):6247-6251.
Bellver, J., Mifsud, A., Grau, N., Privitera, L., \& Meseguer, M. (2013). Similar morphokientic patterns in embryo derived from obese and norm oweight infertile women: a time-lapse study. Hum.Reprod.,28:794-800.

Boomsma, C.M., Eijkemans, M.J., Hughes, E.G., Visser, G.H., Fauser, B.C., \& Macklon, N.S. (2006). A meta-analysis of pregnancy outcomes in women with polycystic ovary syndrome. Hum. Reprod. Update.,12(6): 673-683.

Cakmak, H., \& Taylor, H.S. (2011). Implantation failure: molecular mechanisms and clinical treatment. Hum. Reprod. Update., 1 (2): 242-253.

Cocksedge, K.A., Saravelos, S.H., Wang, Q., Tuckerman, E., Laird, S.M., \& Li, T.C. (2008). Does free androgen index predict subsequent pregnancy outcome in women with recurrent miscarriage? Hum. Reprod., 23:797-802.

Dumesic, D.A., Meldrum, D.R., Katzjaffe, M.G., Krisher, R.L., \& Schoolcraft, W.B. (2015). Oocyte environment: follicular fluid and cumulus cells are critical for oocyte health. Fertil. Steril.,103:303-316.

Duru, S., Bhathena, R.K., \& Safala, S. (2004). The polycystic ovary syndrome. $1^{\text {st }}$ ed. Orient Longman Private Limited.

Fancsovits, P., Murber, Á., Tóthné, G.Z., Rigó, J.J., \& Urbancsek, J. (2011). Human oocytes containing large cytoplasmic vacuoles can result in pregnancy and viable offspring. Reprod. Biomed. Online.,23:513-516.

Fauser, B.C., Tarlatzis, B.C., \& Rebr, R.W. (2012). Consensus on women health aspect of polycystic ovary syndrome (PCOS). The Amesterdam ESHRE/ASRM-sponsered $\quad 3^{\text {rd }}$ PCOS Consensus Workshop Group. Fertil. Stertil.,97(1):28-38.

Fritz, M.A., \& Speroff, L. (2012). Clinical Gynecologic Endocrinology and Infertility. Chronic An ovulation and The Polycystic Ovarian Syndrome. Lippincot W. and Whilkin (eds.) $8^{\text {th }}$ ed. CRC Press Taylor \& Francis Group.,475-531. 
Fulghesu, A.M., Canu. E., Porru, C., \& Cappai, A. (2017). Ultrasound Diagnosis of Polycystic Ovarian Syndrome: Current Guideline, Criticism and Possible Update. Austin J. of Obestet. and Gynecol.,4(2):1074.

Hoffman, B., Schorge, J., Schaffer, J., Lisa, M., Karen, D., \& GaryCunningham, F. (2012). Polycystic ovarian syndrome and hyperandrogenisim. In: Williams Gynecology.2 $2^{\text {nd }} e d$. McGraw-Hill Companies, Inc.,400-606.

Josefin, K., Eliza, C.T., Marie, M., Anju, E.J., Elisabet, S.V., Helena, T., \& Lisa, JM. (2017). Weight Management Interventions in Women with and without PCOS: A Systematic Review. Nutrients.,9:996.

Kini, S. (2012). Polycystic ovary syndrome: diagnosis and management of related infertility. Obstetrics Gynaecology \& Reproductive Medicine., 22:347-35.

Krishna, M., \& Lalorava, M. (2013). Insulin paradox PCOS implication in mechanism and pathogenesis. Health Science., 2(1):j/oo4D.

Mahesh, G., Daneshwar, S., Manju, T., Angelin, P., Soumitra, S., \& Preeti, G. (2018). A cross sectional study of polycystic ovarian syndrome among young women in Bhopal, Central India. Int. J. Community Med. Public Health.,5(1):95-100.

Mina, A. (2013). Morphological expression of human egg and embryo quality. In :Textbook of clinical embryology. Coward $\mathrm{K}$ and Wells D. (eds.). Cambridge University Press.,313326.

Mojgan, M. (2017). Relationship Between Health-Promoting Lifestyle and Quality of Life in Women With Polycystic Ovarian Syndrome. Int. J. of Women's Health and Rep. Sciences.,5(4):318-323.

Okohue, J., Onuh, S., \& Ikimalo, J. (2013). Comparison of IVF/ICSI outcome in patients with polycystic ovarian syndrome or tubal factor infertility. Nigerian J. of Clinical Practice., 16(2):207-210.
Revelli, A., Piane, L.D., Casano, S., Molinari, E., Massobrio, M., \& Rinaudo, P. (2009). Follicular fluid content and oocyte quality from single biochemical markers to metabolomics. Reprod. Biol. Endocrinol.,7:1-13.

Satin, S., \& Bruce, R. (2008). Oocyte quality in adult polycystic ovary syndrome. Semin. Reprod. Med.,26:196-203.

Selda, U., Ahmet, Z.I., Serenat, E., Seyran, Y., Yakup, Y., \& Pelin, O.O. (2015). Correlation of Endometrial Glycodelin Expression and Pregnancy Outcome in Cases with Polycystic Ovary Syndrome Treated with Clomiphene Citrate Plus Metformin: A Controlled Study. Hindawi Publishing Corporation Obstetrics and Gynecology International.,278591,8p.

Stepto, N.K., Cassar, S., Joham, A.E., Hutchison, S.K., Harrison, C.L., Goldstein, R.F. \& Teede, H.J. (2013). Women with polycystic ovary syndrome have intrinsic insulin resistance on euglycaemichyperinsulaemic clamp. Hum. Reprod.,28:777-784.

Sudha, A., Sadanand, B., Patil, Rekha, M., \& Shobha, D. (2017). Role of luteinizing hormone $\mathrm{LH}$ and insulin resistance in polycystic ovary syndrome. Int. J. Repord. Contracept. Obestet. Gyneco.,6(9):3892-3896.

Susie, J., Thomas, H., \& Adam, H. (2018). Polycystic ovary syndrome and assisted reproduction. In : Textbook of assisted reproductive techniques. David K. and Colin M. (eds.). $5^{\text {th }}$ ed. CRC Press Taylor \& Francis Group.,762-772.

Teede, H., Deeks, A., \& Moran, L. (2010). Polycystic ovary syndrome: A complex condition with psychological, reproductive and metabolic manifestations that impacts on health across the lifespan. BMC Med.,8:41.

Walters, K.A., \& Handelsman, D.J. (2016). Androgen receptor splice variants and polycystic ovary syndrome: cause or effect?. Asian J. Androl.,18:442-443. 
Wissing, M.L., Bjerge, M.R., Hoest, T., \& Mikkeilsen, A.L. (2014). Impact of PCOS on early embryo cleavage kinetics. Reproductive BioMedicine online.,18:508514.

Yahia, W., Mahmoud, S., \& Tamer, F. (2013). Prediction of intracytoplasmic sperm injection outcome in patients with polycystic ovary syndrome using follicular anti-mullerian. AAMJ.,10(4)-SUPLL 2.

Yan, Z., Lingyan, L., Tai-Lang, Y., Jing, Y., \& Cheng-Liang, X. (2016). Follicular metabolic changes and effects on oocyte quality in polycystic ovary syndrome patients .Oncotarget.,8(46):80472-80480 